Indian J. Phys. 66A (4), 557 (1992)

\title{
A topological spin-statistics theorem or a use of the antiparticle*
}

\author{
A P Balachandran ${ }^{\dagger}$, A Daughton ${ }^{\dagger}, \mathrm{Z} \mathrm{C} \mathrm{Gu}^{\dagger}, \mathrm{G} \mathrm{Marmo}{ }^{* *}, \mathrm{R}$ D Sorkin ${ }^{\dagger}$, \\ and $A$ M Srivastava ${ }^{\dagger \dagger}$
}

Abstract : A spin-statistics theorem for spinning particles in $R^{d}$ is proved without using relatuvity or field theory, but assuming the existence of antuparucles. The theorem excludes nonabelian statisucs such as parastatusucs of order 2 and more for $d \geq 3$.

* The article has already been published in Mod. Phys. Lett. A5, 1575 (1990).

1 Physics Department, Syracuse University, Syracuse, NY 13244-1130.

** Dipartimento di Scienze Fisıche, Universita dı Napoli and INFN, Scctıon of Naples, Mostra d'Oltremare, Pad. 19, 80125 Napoli, Italy.

$+\dagger$ Theoretical Physics Instutute, University of Minnesota, Minneapolis, Minnesota 55455. 